\title{
Wigner Rotations of Different Types of Lorentz Transformations
}

\author{
A. R. Baizid ${ }^{1,2 *}$, M. S. Alam ${ }^{2}$ \\ ${ }^{1}$ Department of Business Administration, Leading University, Sylhet, Bangladesh \\ ${ }^{2}$ Department of Physics, Shahjalal University of Science and Technology, Sylhet, Bangladesh \\ Received 14 March 2016, accepted in final revised form 21 April 2016
}

\begin{abstract}
We have studied Wigner rotations of different types of Lorentz Transformations according to the nature of movement of one inertial frame relative to the other inertial frame. When the motion is along any arbitrary direction then we can find the formulae for Wigner rotations using the velocity addition formulae for most general, mixed number, quaternion and geometric product Lorentz transformations. Finally we have used simulated data for applying the Wigner rotation formula in pion decay chain and concluded the result.
\end{abstract}

Keywords: Special Lorentz transformation; Most general Lorentz transformation; Mixed number Lorentz transformation; Quaternion Lorentz transformation; Geometric product Lorentz transformation; Wigner Rotation.

๑) 2016 JSR Publications. ISSN: 2070-0237 (Print); 2070-0245 (Online). All rights reserved. doi: http://dx.doi.org/10.3329/jsr.v8i3.27033 J. Sci. Res. 8 (3), 249-258 (2016)

\section{Introduction}

The composition of two Lorentz boosts when they are not collinear results a Lorentz transformation [LT] that is not a pure boost but is the composition of a boost and a rotation. This rotation is called Thomas rotation, Thomas-Wigner rotation or Wigner rotation. The rotation was discovered by Thomas in 1926 and derived by Wigner in 1939 [1]. If a sequence of non-collinear Lorentz transformations returns an object to its initial velocity, then the sequence of Wigner rotations can combine to produce a net rotation called the Thomas precession [2]. The Thomas effect in nuclear spectroscopy is mentioned in Jackson's book on electrodynamics [3]. In fact, the Wigner rotation is the key issue in many branches of physics involving LTs [4].

The Wigner rotation appears in physical processes whose underlying mathematical language includes the Lorentz group; Berry's phase is an example of it [5, 6]. This branch of physics deals with a physical system which gains a phase angle after coming

*orresponding author: sustatik2@gmail.com 
back to the original state at the end of a series of transformations. If the transformations include those of a group isomorphic to the Lorentz group, the Wigner rotation plays a vital role in that case [7].

In recent times the Lorentz group has become an important scientific language in both quantum and classical optics. The theory of squeezed states is a representation of the Lorentz group [8,9]. Optical instruments are everywhere in modern physics based on classical ray optics. It is enjoyable to observe that the Lorentz group is the essential scientific language for ray optics, including polarization optics [10], interferometers [11], lens optics [12,13], laser cavities [14] and multi-layer optics [15].

It is possible to perform mathematical operations of the Lorentz group by arranging optical instruments. For instance, the group contraction is one of the most sophisticated operations in the Lorentz group. Since there are many mathematical operations in Quantum field theory and optical sciences corresponding to LTs, the Wigner rotation becomes one of the important issues in classical and quantum optics. There are different types of LTs. At first we have discussed these LTs.

\subsection{Special Lorentz transformation}

Let us consider two inertial frames of reference $S$ and $S$, where the frame $S$ is at rest and the frame $S^{\prime}$ is moving along the $\mathrm{X}$-axis with velocity $U$ with respect to the $S$ frame. The space and time co-ordinates of $S$ and $S^{\prime}$ are $(\mathrm{x}, \mathrm{y}, \mathrm{z}, \mathrm{t})$ and $\left(\mathrm{x}^{\prime}, \mathrm{y}^{\prime}, \mathrm{z}^{\prime}, \mathrm{t}^{\prime}\right)$ respectively. The relation between the co-ordinates of $S$ and $S$ is called the special Lorentz transformation (SLT), can be written as [16]

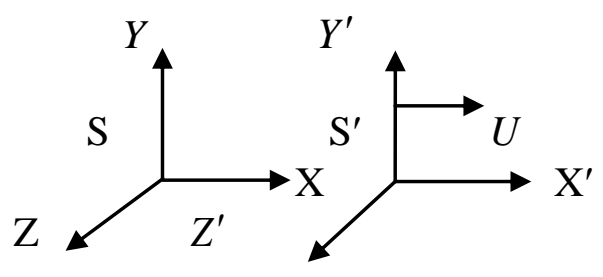

Fig. 1. Special Lorentz transformation

$$
x^{\prime}=\frac{x-U t}{\sqrt{1-U^{2} / c^{2}}}, y^{\prime}=y, z^{\prime}=z, t^{\prime}=\frac{t-U x / c^{2}}{\sqrt{1-U^{2} / c^{2}}}
$$

and the inverse SLT can be written as

$$
x=\frac{x^{\prime}+U t^{\prime}}{\sqrt{1-U^{2} / c^{2}}}, y=y^{\prime}, z=z^{\prime}, t=\frac{t^{\prime}+U x^{\prime} / c^{2}}{\sqrt{1-U^{2} / c^{2}}}
$$




\subsection{Most general Lorentz transformation}

When the motion of the moving frame is along any arbitrary direction instead of $\mathrm{X}$ axis , i.e., the velocity $\vec{U}$ has three components $U_{\mathrm{x}}, U_{\mathrm{y}}$ and $U_{\mathrm{z}}$ then the relation between the space and time co-ordinates of $S$ and $S^{\prime}$ is called the most general Lorentz transformation(MGLT), can be written as [17]

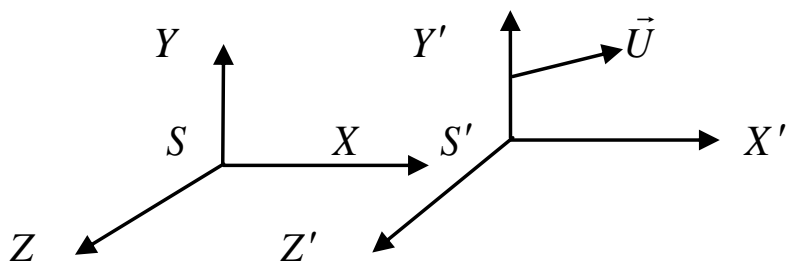

Fig . 2. Most general Lorentz transformation

$\vec{r}^{\prime}=\vec{r}+\vec{U}\left\{\frac{(\vec{r} \cdot \vec{U})}{U^{2}}(\gamma-1)-t \gamma\right\}$

$t^{\prime}=\gamma\{t-\vec{r} \cdot \vec{U}\}$

and the inverse MGLT can be written as

$\vec{r}=\vec{r}^{\prime}+\vec{U}\left\{\frac{\left(\vec{r}^{\prime} \cdot \vec{U}\right)}{U^{\prime 2}}(\gamma-1)+t^{\prime} \gamma\right\}$

$t=\gamma\left(t^{\prime}+\vec{r}^{\prime} \cdot \vec{U}^{\prime}\right)$

where, $\quad \gamma=\left(1-\frac{U^{2}}{c^{2}}\right)^{-\frac{1}{2}}, \vec{r}=x \widehat{i}+y \widehat{j}+z \hat{k}, \vec{r}^{\prime}=x^{\prime} \widehat{i}+y^{\prime} \widehat{j}+z^{\prime} \widehat{k}, c=1$

\section{3. Mixed number Lorentz transformation}

Consider same case as MGLT, then using the mixed product [1820] $\vec{A} \otimes \vec{B}=\vec{A} \cdot \vec{B}+i \vec{A} \times \vec{B}$, the mixed number Lorentz transformation [21] (MNLT) can be written as

$t^{\prime}=\gamma(t-\vec{r} \cdot \vec{U})$

$\vec{r}^{\prime}=\gamma(\vec{r}-t \vec{U}-i \vec{r} \times \vec{U})$

and the inverse MNLT can be written as

$t=\gamma\left(t^{\prime}+\vec{r}^{\prime} \cdot \vec{U}\right)$

$\vec{r}=\gamma\left(\vec{r}^{\prime}+t^{\prime} \vec{U}+i \vec{r}^{\prime} \times \vec{U}\right)$ 


\subsection{Quaternion Lorentz, transformation}

Again, consider same case as MGLT, then using the quaternion product [22-24] $\underline{\vec{A} \vec{B}}=-\vec{A} \cdot \vec{B}+\vec{A} \times \vec{B}$ the quaternion Lorentz transformation (QLT) [25] can be written as

$t^{\prime}=\gamma(t+\vec{r} \cdot \vec{U})$

$\vec{r}^{\prime}=\gamma(\vec{r}-t \vec{U}-\vec{r} \times \vec{U})$

and the inverse QLT, can be written as

$t=\gamma\left(t^{\prime}-\vec{r}^{\prime} \cdot \vec{U}\right)$

$\vec{r}=\gamma\left(\vec{r}^{\prime}+t^{\prime} \vec{U}+\vec{r}^{\prime} \times \vec{U}\right)$

\subsection{Geometric Product Lorentz transformation}

Again, consider same case as MGLT, then using the geometric product of two vectors [26-28] $\vec{A} \vec{B}=\vec{A} \cdot \vec{B}+\vec{A} \times \vec{B}$ the geometric product Lorentz transformation [21] (GPLT) can be written as

$t^{\prime}=\gamma(t-\vec{r} \cdot \vec{U})$

$\vec{r}^{\prime}=\gamma(\vec{r}-t \vec{U}-\vec{r} \times \vec{U})$

and the inverse GPLT can be written as

$t=\gamma\left(t^{\prime}+\vec{r}^{\prime} \cdot \vec{U}\right)$

$\vec{r}=\gamma\left(\vec{r}^{\prime}+t^{\prime} \vec{U}+\vec{r}^{\prime} \times \vec{U}\right)$

\section{Wigner Rotation}

Consider the pion decay chain $\pi \rightarrow \mu \rightarrow e^{-}$, where pion $(\pi)$ is moving with velocity $\vec{U}$ with respect to lab frame $S$, muon $(\mu)$ is moving with velocity $\vec{V}$ with respect to $\pi$, electron $(e)$ is moving with velocity $\vec{W}$ with respect to $\mu$ then we want to find the velocity of electron with respect to lab frame in different types of LTs. There are two ways to get the velocity of electron with respect to lab frame $(\vec{U} \oplus \vec{V}) \oplus \vec{W}$ and $\vec{U} \oplus(\vec{V} \oplus \vec{W})$. The angle between these two velocity vectors is called Wigner rotation, where $\oplus$ denotes the Lorentz sum. 

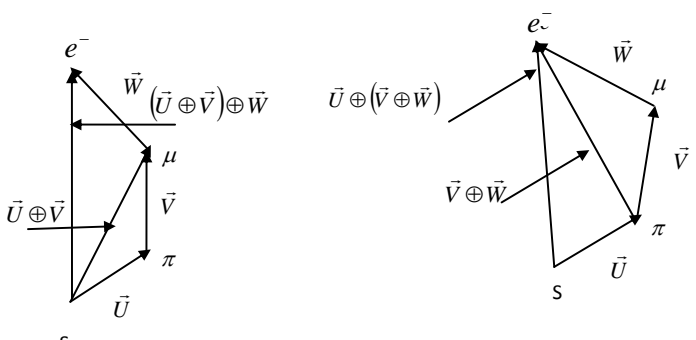

Fig. 3. Wigner rotation of Lorentz transformations.

\subsection{Wigner Rotation for special Lorentz transformation}

SLT is one dimensional. The velocity of the moving frame is along $\mathrm{x}$-axis. So there is no Wigner rotation for SLT.

\subsection{Wigner Rotation for most general Lorentz transformation}

If $\vec{W}^{\prime \prime}$ be the velocity of muon with respect to lab frame then according to the velocity addition formula for the MGLT [21] we can write

$$
\vec{W}^{\prime \prime}=\vec{U} \oplus \vec{V}=\frac{\vec{V}+\vec{U}\left[(\vec{U} \cdot \vec{V}) / U^{2}\left(\gamma_{U}-1\right)+\gamma_{U}\right]}{\gamma_{U}(1+\vec{U} \cdot \vec{V})}
$$

Now, if muon moves with velocity $\vec{W}^{\prime \prime}$ with respect to lab frame and electron moves with velocity $\vec{W}$ respect to muon then according to the velocity addition formula for MGLT [21] we can write

$$
\begin{aligned}
& \vec{W}^{\prime \prime \prime}=\vec{W}^{\prime \prime} \oplus \vec{W}=\frac{\vec{W}+\vec{W}^{\prime \prime}\left\{\frac{\left(\vec{W}^{\prime \prime} \cdot \vec{W}\right)}{W^{\prime 2}}\left(\gamma_{\vec{W}^{\prime \prime}}-1\right)+\gamma_{\vec{W}^{\prime \prime}}\right\}}{\gamma_{\vec{W}^{\prime \prime}}\left(1+\vec{W}^{\prime \prime} \cdot \vec{W}\right)} \\
& o r,(\vec{U} \oplus \vec{V}) \oplus \vec{W}=\frac{\vec{W}+(\vec{U} \oplus \vec{V})\left\{\frac{(\vec{U} \oplus \vec{V}) \cdot \vec{W}}{(\vec{U} \oplus \vec{V})^{2}}\left(\gamma_{(\vec{U} \oplus \vec{V})}-1\right)+\gamma_{(\vec{U} \oplus \vec{V})}\right\}}{\gamma_{(\vec{U} \oplus \vec{V})}(1+(\vec{U} \oplus \vec{V}) \cdot \vec{W})}
\end{aligned}
$$

Again, from muon and electron according to the velocity addition formula for MGLT [21] the resultant velocity of $\vec{V}$ and $\vec{W}$ can be written as

$$
\vec{W}^{\prime}=\vec{V} \oplus \vec{W}=\frac{\vec{W}+\vec{V}\left\{\frac{(\vec{V} \cdot \vec{W})}{V^{2}}\left(\gamma_{V}-1\right)+\gamma_{V}\right\}}{\gamma_{V}(1+\vec{V} \cdot \vec{W})}
$$




\section{Wigner Rotations}

Finally, if electron moves with velocity $\vec{W}^{\prime}$ with respect to pion then the resultant velocity of electron with respect to lab frame can be written as

$$
\begin{aligned}
& \vec{W}^{\prime \prime \prime}=\vec{U} \oplus \vec{W}^{\prime}=\frac{\vec{W}^{\prime}+\vec{U}\left\{\frac{\left(\vec{U} \cdot \vec{W}^{\prime}\right)}{U^{2}}\left(\gamma_{U}-1\right)+\gamma_{U}\right\}}{\gamma_{U}\left(1+\vec{U} \cdot \vec{W}^{\prime}\right)} \\
& \text { or, } \vec{U} \oplus(\vec{V} \oplus \vec{W})=\frac{(\vec{V} \oplus \vec{W})+\vec{U}\left\{\frac{\vec{U} \cdot(\vec{V} \oplus \vec{W})}{U^{2}}\left(\gamma_{U}-1\right)+\gamma_{U}\right\}}{\gamma_{U}(1+(\vec{V} \oplus \vec{W}) \cdot \vec{U})}
\end{aligned}
$$

Specifically to illustrate Wigner rotation we have used simulated data for velocity vectors in unit of $c$, defined as

$\vec{U}=\left(u_{x}, u_{y}, 0\right)=(0.5,0.2,0.0)$ velocity of pion relative to lab frame;

$\vec{V}=\left(v_{x}, v_{y}, 0\right)=(0.3,0.5,0.0)$ velocity of muon relative to pion ;

$\vec{W}=\left(w_{x}, w_{y}, 0\right)=(0.4,0.2,0.0$,$) velocity of electron relative to muon.$

The corresponding $\gamma$ factors are as follows:

$$
\gamma_{U}=\frac{1}{\sqrt{1-U^{2}}}=1.18678, \gamma_{V}=\frac{1}{\sqrt{1-V^{2}}}=1.230915, \gamma_{(\vec{U} \oplus \vec{V})}=\frac{1}{\sqrt{1-(\vec{U} \oplus \vec{V})^{2}}}=1.826029
$$

From equation (11) and (12) we have $(\vec{U} \oplus \vec{V}) \oplus \vec{W}=\left[\begin{array}{c}0.7545323 \\ 0.549697 \\ 0\end{array}\right]=\vec{A}$ (say)

From equation (13) and (14) we get $\vec{U} \oplus(\vec{V} \oplus \vec{W})=\left[\begin{array}{c}.7646265 \\ .5337916 \\ 0\end{array}\right]=\vec{B}$ (say)

We know that

$$
\begin{gathered}
\vec{A} \cdot \vec{B}=A B \cos \theta \\
\text { or }, \theta=\cos ^{-1}\left(\frac{\vec{A} \cdot \vec{B}}{A B}\right)
\end{gathered}
$$

where

$$
\vec{A}=0.7545323 \hat{i}+0.549697 \hat{j}+0 \hat{k}, A=\sqrt{(0.7545323)^{2}+(0.549697)^{2}}=.933534
$$

and

$$
\vec{B}=0.7646265 \hat{i}+0.5337916 \hat{j}+0 \hat{k}, B=\sqrt{(0.7646265)^{2}+(0.5337916)^{2}}=.9325166
$$

\section{$\vec{A} \cdot \vec{B}=.870359032$}

Hence, $\theta_{\text {Wigner }}=\cos ^{-1}(.99979)=1.15^{0}$

Again let, 
$\vec{U}=\left(u_{x}, u_{y}, 0\right)=(0.5,0.2,0.0), \vec{V}=\left(v_{x}, v_{y}, 0\right)=(0.2,0.4,0.0), \vec{W}=\left(w_{x}, w_{y}, 0\right)=(0.5,0.3,0.0) \&$

$\vec{U}=\left(u_{x}, u_{y}, 0\right)=(0.4,0.3,0.0), \vec{V}=\left(v_{x}, v_{y}, 0\right)=(0.2,0.5,0.0), \vec{W}=\left(w_{x}, w_{y}, 0\right)=(0.6,0.3,0.0)$

be two sets of velocity vectors of pion decay chain $\pi \rightarrow \mu \rightarrow e^{-}$, as Fig. 3 then using equations (11),(12),(13) and (14) we have the velocity vectors of electron relative to lab frame, $(\vec{U} \oplus \vec{V}) \oplus \vec{W}$ and $\vec{U} \oplus(\vec{V} \oplus \vec{W})$ are

$\left[\begin{array}{c}0.755839 \\ 0.547582 \\ 0\end{array}\right],\left[\begin{array}{c}0.796143 \\ 0.4592595 \\ 0\end{array}\right]$ and $\left[\begin{array}{c}0.68265 \\ 0.668553 \\ 0\end{array}\right],\left[\begin{array}{c}0.69576 \\ 0.653219 \\ 0\end{array}\right]$ respectively.

Using similar process as previous one we have the Wigner rotations in these cases $\theta_{\text {Wigner }}=\cos ^{-1}(0.9946244)=5.94^{\circ}$ (App.) and $\theta_{\text {Wigner }}=\cos ^{-1}(0.999778)=1.2^{\circ} \quad$ (App.) respectively.

\subsection{Wigner Rotation of mixed number Lorentz transformation}

If $\vec{W}^{\prime \prime}$ be the velocity of muon with respect to lab frame then according to the velocity addition formula for the MNLT [21] we can write

$\vec{W}^{\prime \prime}=\vec{U} \oplus \vec{V}=\frac{\vec{U}+\vec{V}+i \vec{V} \times \vec{U}}{1+\vec{U} \cdot \vec{V}}$

Now using similar process as MGLT the velocity vectors of electron relative to lab frame for MNLT as Fig. 3, we have

$$
\begin{aligned}
& (\vec{U} \oplus \vec{V}) \oplus \vec{W}=\frac{(\vec{U} \oplus \vec{V})+\vec{W}+i \vec{W} \times(\vec{U} \oplus \vec{V})}{1+(\vec{U} \oplus \vec{V}) \vec{W}} \\
& \vec{U} \oplus(\vec{V} \oplus \vec{W})=\frac{\vec{U}+(\vec{V} \oplus \vec{W})+i(\vec{V} \oplus \vec{W}) \times \vec{U}}{1+\vec{U} \cdot(\vec{V} \oplus \vec{W})}
\end{aligned}
$$

and

Specifically to illustrate Wigner rotation for MNLT velocity vectors are defined as $\vec{U}=\left(u_{x}, u_{y}, 0\right)=(0.5,0.2,0.0), \vec{V}=\left(v_{x}, v_{y}, 0\right)=(0.3,0.5,0.0), \vec{W}=\left(w_{x}, w_{y}, 0\right)=(0.4,0.2,0.0$,$) ,$ $\vec{U}=\left(u_{x}, u_{y}, 0\right)=(0.5,0.2,0.0), \vec{V}=\left(v_{x}, v_{y}, 0\right)=(0.2,0.4,0.0), \vec{W}=\left(w_{x}, w_{y}, 0\right)=(0.5,0.3,0.0) \&$ $\vec{U}=\left(u_{x}, u_{y}, 0\right)=(0.4,0.3,0.0), \vec{V}=\left(v_{x}, v_{y}, 0\right)=(0.2,0.5,0.0), \vec{W}=\left(w_{x}, w_{y}, 0\right)=(0.6,0.3,0.0)$

be three sets of velocity vectors of pion decay chain $\pi \rightarrow \mu \rightarrow e^{-}$, as Fig. 3 then using equations (15), (16) and (17) we have the velocity vectors of electron relative to lab frame are $(\vec{U} \oplus \vec{V}) \oplus \vec{W}$ and $\vec{U} \oplus(\vec{V} \oplus \vec{W})$ are

$$
\left[\begin{array}{c}
0.94 \\
0.64 \\
-.058 i
\end{array}\right],\left[\begin{array}{c}
0.88 \\
0.62 \\
-.055 i
\end{array}\right],\left[\begin{array}{c}
0.7825 \\
0.5111 \\
-.0409 i
\end{array}\right],\left[\begin{array}{c}
0.7825 \\
0.5111 \\
-.0409 i
\end{array}\right] \&\left[\begin{array}{l}
0.70819 \\
0.6847 \\
0.2404 i
\end{array}\right],\left[\begin{array}{l}
0.7541 \\
0.59288 \\
0.08535 i
\end{array}\right] \text { respectively. }
$$


Using similar process as MGLT we have the Wigner rotations of each case $\theta_{\text {Wigner }}=\cos ^{-1}(1)=0^{\circ}$

\subsection{Wigner Rotation of Quaternion Lorentz transformation}

If $\vec{W}^{\prime \prime}$ be the velocity of muon with respect to lab frame then according to the velocity addition formula for QLT [29] we can write

$$
\vec{W}^{\prime \prime}=\vec{U} \oplus \vec{V}=\frac{\vec{U}+\vec{V}+\vec{V} \times \vec{U}}{1-\vec{U} \cdot \vec{V}}
$$

Now using similar process as MGLT the velocity vectors of electron relative to lab frame $S$ for QLTas Fig. 3, we have

$$
\begin{aligned}
& (\vec{U} \oplus \vec{V}) \oplus \vec{W}=\frac{(\vec{U} \oplus \vec{V})+\vec{W}+\vec{W} \times(\vec{U} \oplus \vec{V})}{1-(\vec{U} \oplus \vec{V}) \cdot \vec{W}} \\
& \vec{U} \oplus(\vec{V} \oplus \vec{W})=\frac{\vec{U}+(\vec{V} \oplus \vec{W})+(\vec{V} \oplus \vec{W}) \times \vec{U}}{1-\vec{U} \cdot(\vec{V} \oplus \vec{W})}
\end{aligned}
$$

Specifically to illustrate Wigner rotation for QLT velocity vectors are defined as

$$
\begin{aligned}
& \vec{U}=\left(u_{x}, u_{y}, 0\right)=(0.5,0.2,0.0), \vec{V}=\left(v_{x}, v_{y}, 0\right)=(0.3,0.5,0.0), \vec{W}=\left(w_{x}, w_{y}, 0\right)=(0.4,0.2,0.0,), \\
& \vec{U}=\left(u_{x}, u_{y}, 0\right)=(0.5,0.2,0.0), \vec{V}=\left(v_{x}, v_{y}, 0\right)=(0.2,0.4,0.0), \vec{W}=\left(w_{x}, w_{y}, 0\right)=(0.5,0.3,0.0) \& \\
& \vec{U}=\left(u_{x}, u_{y}, 0\right)=(0.4,0.3,0.0), \vec{V}=\left(v_{x}, v_{y}, 0\right)=(0.2,0.5,0.0), \vec{W}=\left(w_{x}, w_{y}, 0\right)=(0.6,0.3,0.0)
\end{aligned}
$$

be three sets of velocity vectors of pion decay chain $\pi \rightarrow \mu \rightarrow e^{-}$, as Fig. 3 then using equations (18), (19) and (20) we have the velocity vectors of electron relative to lab frame $\operatorname{are}(\vec{U} \oplus \vec{V}) \oplus \vec{W}$ and $\vec{U} \oplus(\vec{V} \oplus \vec{W})$;

$$
\left[\begin{array}{l}
3.662 \\
3.193 \\
-0.241
\end{array}\right],\left[\begin{array}{l}
3.662 \\
3.193 \\
-0.241
\end{array}\right],\left[\begin{array}{c}
3.6278 \\
3.167 \\
-0.2388
\end{array}\right],\left[\begin{array}{c}
3.6586 \\
3.191 \\
-0.2395
\end{array}\right] \&\left[\begin{array}{l}
5.999 \\
5.558 \\
0.9411
\end{array}\right],\left[\begin{array}{l}
5.999 \\
5.558 \\
0.9411
\end{array}\right] \text { respectively. }
$$

Using similar process as MGLT we have the Wigner rotations of each case $\theta_{\text {Wigner }}=\cos ^{-1}(1)=0^{\circ}$

\subsection{Wigner Rotation of Geometric product Lorentz transformation}

If $\vec{W}^{\prime \prime}$ be the velocity of muon with respect to lab frame then according to the velocity addition formula for GPLT [21] we can write

$$
\vec{W}^{\prime \prime}=\vec{U} \oplus \vec{V}=\frac{\vec{U}+\vec{V}+\vec{V} \times \vec{U}}{1+\vec{U} \cdot \vec{V}}
$$

Now using similar process the velocity vectors of electron relative to lab frame for GPLTas Fig. 3, we have 


$$
\begin{aligned}
& (\vec{U} \oplus \vec{V}) \oplus \vec{W}=\frac{(\vec{U} \oplus \vec{V})+\vec{W}+\vec{W} \times(\vec{U} \oplus \vec{V})}{1+(\vec{U} \oplus \vec{V}) \cdot \vec{W}} \\
& \vec{U} \oplus(\vec{V} \oplus \vec{W})=\frac{\vec{U}+(\vec{V} \oplus \vec{W})+(\vec{V} \oplus \vec{W}) \times \vec{U}}{1+\vec{U} \cdot(\vec{V} \oplus \vec{W})}
\end{aligned}
$$

and

Specifically to illustrate Wigner rotations for GPLTvelocity vectors are defined as

$$
\begin{aligned}
& \vec{U}=\left(u_{x}, u_{y}, 0\right)=(0.5,0.2,0.0), \vec{V}=\left(v_{x}, v_{y}, 0\right)=(0.3,0.5,0.0), \vec{W}=\left(w_{x}, w_{y}, 0\right)=(0.4,0.2,0.0,), \\
& \vec{U}=\left(u_{x}, u_{y}, 0\right)=(0.5,0.2,0.0), \vec{V}=\left(v_{x}, v_{y}, 0\right)=(0.2,0.4,0.0), \vec{W}=\left(w_{x}, w_{y}, 0\right)=(0.5,0.3,0.0) \& \\
& \vec{U}=\left(u_{x}, u_{y}, 0\right)=(0.4,0.3,0.0), \vec{V}=\left(v_{x}, v_{y}, 0\right)=(0.2,0.5,0.0), \vec{W}=\left(w_{x}, w_{y}, 0\right)=(0.6,0.3,0.0)
\end{aligned}
$$

be three sets of velocity vectors of pion decay chain $\pi \rightarrow \mu \rightarrow e^{-}$, as Fig. 3 then using equations (21), (22) and (23) we have the velocity vectors of electron relative to lab frame are $(\vec{U} \oplus \vec{V}) \oplus \vec{W}$ and $\vec{U} \oplus(\vec{V} \oplus \vec{W})$;

$$
\left[\begin{array}{l}
0.738 \\
0.6 \\
-0.0409
\end{array}\right],\left[\begin{array}{l}
0.7497 \\
0.59298 \\
-0.040936
\end{array}\right],\left[\begin{array}{c}
0.726316 \\
0.604678 \\
-0.040936
\end{array}\right],\left[\begin{array}{c}
0.7497073 \\
0.5929822 \\
-0.040936
\end{array}\right] \&\left[\begin{array}{l}
0.708196 \\
0.6847 \\
0.08743
\end{array}\right],\left[\begin{array}{l}
0.67541 \\
0.6978134 \\
0.087433
\end{array}\right]
$$

respectively.

Using similar process as MGLT we have the Wigner rotations of the above cases are $\theta_{\text {Wigner }}=\cos ^{-1}(.9999171)=0.74^{\circ}, \theta_{\text {Wigner }}=\cos ^{-1}(.99259)=2.2^{\circ}$ $\& \theta_{\text {Wigner }}=\cos ^{-1}(0.99945)=1.9^{\circ}$ respectively.

\section{Comparison of the Study}

3.1. Comparison of Wigner Rotations of special, most general, mixed number, quaternion and geometric product Lorentz transformations

\begin{tabular}{llll}
\hline $\begin{array}{l}\text { Names of } \\
\text { Lorentz }\end{array}$ & Wigner Rotation & Wigner Rotation & Wigner Rotation \\
transformations & $\vec{U}=\left(u_{x}, u_{y}, 0\right)=(.5, .2,0)$ & $\vec{U}=\left(u_{x}, u_{y}, 0\right)=(.5,2,0)$ & $\vec{U}=\left(u_{x}, u_{y}, 0\right)=(.4,3,0)$ \\
& $\vec{V}=\left(v_{x}, v_{y}, 0\right)=(.3, .5,0)$ & $\vec{V}=\left(v_{x}, v_{y}, 0\right)=(.2, .4,0)$ & $\vec{V}=\left(v_{x}, v_{y}, 0\right)=(.2, .5,0)$ \\
& $\vec{W}=\left(w_{x}, w_{y}, 0\right)=(.4,2,0)$ & $\vec{W}=\left(w_{x}, w_{y}, 0\right)=(.5,3,0)$, & $\vec{W}=\left(w_{x}, w_{y}, 0\right)=(.6,3,0)$, \\
& & & \\
\hline SLT & Not applicable & Not applicable & Not applicable \\
MGLT & $1.15^{\circ}$ & $5.94^{\circ}$ & $1.2^{\circ}$ \\
MNLT & $0^{\circ}$ & $0^{\circ}$ & $0^{\circ}$ \\
QLT & $0^{\circ}$ & $0^{\circ}$ & $0^{\circ}$ \\
GPLT & $0.74^{\circ}$ & $2.2^{\circ}$ & $1.9^{\circ}$ \\
\hline
\end{tabular}

\section{Conclusion}

We have discussed the Wigner rotations for different types of LTs. In the case of MGLT and GPLT we have found Wigner rotations but the values are different in each 
case. In the case of MNLT and QLT, there is no Wigner Rotation. We can apply our results in the study of hidden magnetic forces manifest in some problems of Electromagnetism, Spin-orbit interaction of electron with nucleus in an atom in Quantum Mechanics, study of the quantized electromagnetic field in phase space and of the interaction between atoms and photons in cavities in quantum optics.

\section{References}

1. E. P. Wigner, Ann. Math. 40, 149 (1939). http://dx.doi.org/10.2307/1968551

2. H. Kroemer, Am. J. Phys. 72, 51 (2004).

3. J. D. Jackson, Classical Electrodynamics, 3rd edition (Wiley, New York, 1999).

4. N. J. Papastamatiou, H. Matsumoto, and H. Umezawa, Prog. Theoret. Phys. 69(5) 1647 (1983).

5. S. Pancharatnam, Generalized Theory of Interference and its Application - Proc. Indian Acad. Sci. A 44, 247 (1956).

6. M. Berry, J. Mod. Opt. A 34, 1401 (1987). http://dx.doi.org/10.1080/09500348714551321

7. R. Y. Chiao and T. F. Jordan, Phys. Lett. A 132, 77 (1988). http://dx.doi.org/10.1016/03759601(88)90255-1

8. H. P. Yuen, Phys. Rev. A 13, 2226 (1976). http://dx.doi.org/10.1103/PhysRevA.13.2226

9. Y. S. Kim and M. E. Noz, Two Different Squeeze Transformations (World Scientific, Singapore, 1991).

10. D. Han, Y. S. Kim, and M. E. Noz, Phys. Rev. E 56, 6065 (1997). http://dx.doi.org/10.1103/PhysRevE.56.6065

11. D. Han, Y. S. Kim, and M. E. Noz, Phys. Rev. E 61, 5907 (2000). http://dx.doi.org/10.1103/PhysRevE.61.5907

12. E. C. G. Sudarshan, N. Mukunda, and R. Simon, Optica Acta 32, 855 (1985). http://dx.doi.org/10.1080/713821807

13. S. Baskal and Y. S. Kim, J. Opt. Soc. Am. A 26(9), 2049 (2009).

14. S. Baskal and Y. S. Kim, Phys. Rev. E 66, 026604 (2002). http://dx.doi.org/10.1103/PhysRevE.66.026604

15. J. J. Monzon and L. L. Sanchez-Soto, Am. J. Phys. 64, 156 (1996). http://dx.doi.org/10.1119/1.18135

16. R. Resnick, Introduction to Special Relativity (Wiley Eastern limited, 1994).

17. C. Moller, The Theory of Relativity (Oxford University press, London, 1972).

18. M. S Alam, Study of Mixed Number - Proc. Pak. Acad. Sci. 37(1), 119 (2000).

19 M. S. Alam, J. Theoretics 3(4), 1(2001).

20. M. S. Alam, Ind. J. Phys. A 77, 47 (2003).

21. M.S. Alam and K. Begum, Jahangirnagar Phys. Studies 15, 89 (2009).

22. A. Kyrala, Theoretical Physics (W. B. Saunders Company, Philadelphia \& London, 1967).

23. Quaternion (2016). http://mathworld.wolfram.com/Quaternion.html

24. Dr. Sarah's Quaternion Demo (2016). http://www.cs.appstate.edu/ sjg/class/3110/mathfestalg2000/quaternions1.html

25. M. S. Alam and S. Bauk, Quaternion Lorentz Transformation, Physics Essays (American Institute of Physics, Canada, 2011).

26. M. S. Alam, News Bull. Cal. Math. Soc. 26(1-3), 21 (2003).

27. B. K. Datta, V. De Sabbata, and L. Ronchetti, Il Nuovo Cimento, 113B, 711 (1998).

28. B. K. Datta and R. Datta, Found. Phys. Lett. 11, 1 (1998).

29. M. S. Alam and M. D. Chowdhury, J. Nat. Sci. Found. Sri Lanka 34(3), 143 (2006). 